\title{
RNA-bound PGC-1 $\alpha$ controls gene expression in liquid-like nuclear condensates
}

Joaquín Pérez-Schindler ${ }^{1, *}$, Bastian Kohl ${ }^{1}$, Konstantin Schneider-Heieck ${ }^{1}$, Volkan Adak ${ }^{1}$, Julien Delezie ${ }^{1}$, Geraldine Maier ${ }^{1}$, Thomas Sakoparnig ${ }^{1}$, Elyzabeth Vargas-Fernández ${ }^{1}$, Bettina Karrer-Cardel ${ }^{1}$, Danilo Ritz ${ }^{1}$, Alexander Schmidt ${ }^{1}$, Maria Hondele ${ }^{1}$, Sebastian Hiller ${ }^{1}$ \& Christoph Handschin ${ }^{1, *}$

${ }^{1}$ Biozentrum, University of Basel, Basel, Switzerland. *co-corresponding authors.

e-mail: j.perezschindler@unibas.ch; christoph.handschin@unibas.ch 


\section{Abstract}

The peroxisome-proliferator-activated receptor- $\gamma$ coactivator-1 $\alpha$ (PGC-1 $\alpha)$ integrates environmental cues by controlling complex transcriptional networks in various metabolically active tissues. However, it is unclear how a transcriptional coregulator coordinates dynamic biological programs in response to multifaceted stimuli such as endurance training or fasting. Here, we discovered a central function of the poorly understood C-terminal domain (CTD) of PGC-1 $\alpha$ to bind RNAs and assemble multi-protein complexes. Surprisingly, in addition to controlling the coupling of transcription and processing of target genes, RNA binding is indispensable for the recruitment of PGC-1 $\alpha$ to chromatin into liquid-like nuclear condensates, which compartmentalize and regulate active transcription. These results demonstrate a hitherto unsuspected molecular mechanism by which complexity in the regulation of large transcriptional networks by PGC-1 $\alpha$ is achieved. These findings are not only essential for the basic understanding of transcriptional coregulator-driven control of biological programs, but will also help to devise new strategies to modulate these processes in pathological contexts in which PGC-1 $\alpha$ function is dysregulated, such as type 2 diabetes, cardiovascular diseases or skeletal muscle wasting.

\section{Main}

PGC-1 $\alpha$ controls complex biological processes by regulating transcriptional networks sensitive to environmental cues such as fasting in liver, cold in brown adipose tissue and exercise in skeletal muscle ${ }^{1-3}$. Indeed, skeletal muscle-specific overexpression of PGC-1 $\alpha$ in mice induces an endurance trained-like phenotype, characterised by robust mitochondrial biogenesis and enhanced exercise performance ${ }^{1,4}$. The molecular underpinnings that enable such a broad transcriptional control in a spatiotemporal manner however are unclear. PGC-1 $\alpha$ lacks any discernible enzymatic activity and its function is thought to depend on the formation 
of a multi-protein complex containing a multitude of different transcription factors (TFs) and additional transcriptional regulators ${ }^{5,6}$. PGC-1 $\alpha$ is highly conserved from mouse to human with $94 \%$ identity. The protein is intrinsically disordered for the large majority of its 797 amino acids and contains only a single folded domain, an RNA recognition motif (RRM) (Fig. 1a, Fig. 4c). Three sequence features are present in the intrinsically disordered part, an LXXLL motif, typically involved in nuclear receptor binding ${ }^{7}$, and two arginine-serine-rich (RS) regions. The segment of residues 564-797, comprising the two RS and the RRM, is generally termed the C-terminal domain (CTD). Deletion of the CTD of PGC-1 $\alpha$ blunts its positive effects on gene expression, which was originally attributed to impaired RNA processing ${ }^{8,9}$. A direct coupling of transcription to RNA processing was underlined by PGC-1 $\alpha$ interactions with splicing factors and components of the RNA polymerase 2 ( $\mathrm{Pol} 2$ ) complex via the CTD and a mini-gene splicing assay ${ }^{8}$. However, the impact of PGC- $1 \alpha$ on RNA binding and processing of target genes and, hence, the functional relevance of the CTD remain unclear.

\section{PGC-1 $\alpha$ function is impaired by deletion of the CTD}

To investigate the function of the PGC- $1 \alpha$ CTD, we overexpressed either full length (FL) PGC$1 \alpha$ or a truncated protein lacking the CTD $(\Delta \mathrm{CTD})$ in C2C12 myotubes. Both PGC-1 $\alpha$ FL and $\triangle \mathrm{CTD}$ exhibited comparable levels of overexpression at the RNA and protein level in a context of very low expression of endogenous PGC-1 $\alpha$ (Supplementary Fig. 1a, b). Moreover, deletion of the CTD of PGC-1 $\alpha$ did not affect protein half-life compared to FL protein (Supplementary Fig. 1c). In agreement with published data, transcriptome analysis revealed a large number of differentially expressed genes (DEG) upon overexpression of PGC-1 $\alpha$ FL, whereas overexpression of $\triangle \mathrm{CTD}$ induces a drastically different set of DEG (Fig. 1b, Supplementary Fig. 2a). Based on Gene Ontology (GO) analysis, the vast majority of DEG regulated by both PGC-1 $\alpha$ FL and $\triangle$ CTD were related to metabolic pathways (Supplementary Fig. 2b), while the 
function of the $\triangle \mathrm{CTD}$-dependent DEG and those that are exclusively controlled by the CTD is less clearly defined (Supplementary Fig. 2b). We next leveraged our transcriptomic data to infer TFs regulated in a PGC-1 $\alpha$ CTD-dependent manner by using Integrated System for Motif Activity Response Analysis (ISMARA) ${ }^{10}$. This analysis strongly suggests that the CTD of PGC-1 $\alpha$ plays a central role in modulating the activity of a wide range of TFs (Fig. 1c). Interestingly, PGC-1 $\alpha$-induced activation of the nuclear receptor ERR $\alpha$, known to bind at the N-terminal domain, was also predicted to be blunted in PGC-1 $\alpha \Delta$ CTD by ISMARA analysis, suggesting that CTD-specific features and mechanisms play a critical regulatory function.

The biological impact of the deletion of PGC-1 $\alpha$ CTD was further investigated via whole proteome analysis of skeletal muscle cells. Proteome remodelling mediated by PGC-1 $\alpha$ FL was reduced in PGC-1 $\alpha \Delta \mathrm{CTD}$, though the effect was milder compared to that observed at the transcriptome level (Supplementary Fig. 2c, d). Next, we defined a set of "core proteins" by integrating the transcriptomic and proteomic datasets, revealing genes transcriptionally up- or down-regulated by PGC-1 $\alpha$ that exhibit a corresponding change at the protein level. We found that deletion of the CTD of PGC-1 $\alpha$ drastically impaired the expression of such core proteins, which are primarily involved in transport processes and oxidative metabolism (Fig. 1d, e). Importantly, even though many core proteins in the overlap between PGC- $1 \alpha$ FL and $\Delta \mathrm{CTD}$ are also involved in oxidative phosphorylation and tricarboxylic acid cycle (Fig. 1e, Supplementary Fig. 2e, f), only overexpression of PGC-1 $\alpha$ FL significantly increased basal oxygen consumption of skeletal muscle cells (Fig. 1f). Altogether, these data demonstrate that both the transcriptional and functional output of PGC-1 $\alpha$ are highly modulated by the CTD.

\section{The PGC-1 $\alpha$ CTD mediates RNA-dependent protein-protein interactions}

Mechanistically, the CTD of PGC-1 $\alpha$ represents a potential platform for protein-protein and protein-RNA interactions. By implementing an in vitro affinity purification followed by mass 
spectrometry (in vitro AP-MS) assay, we identified over 200 nuclear proteins interacting directly or indirectly with the CTD of PGC-1 $\alpha$ (Fig. 2a). Most of PGC-1 $\alpha$ CTD-interacting proteins were associated with the regulation of RNA processing and gene transcription (Fig. 2b). Consistently, analysis of our transcriptomic data demonstrated that PGC-1 $\alpha$ FL regulates RNA processing events (Fig. 2c), corresponding to the alternative splicing of 203 transcripts (Fig. 2d). In contrast, over $60 \%$ of the alternative splicing capacity was lost when the CTD of PGC-1 $\alpha$ was deleted (Fig. 2c, d). These data support and further expand previous findings proposing a role of PGC-1 $\alpha$ in RNA processing via CTD-mediated protein-protein interactions $^{8,11}$. It is however unknown to what extent direct RNA binding to the CTD of PGC$1 \alpha$ is involved in this process. We therefore carried out in vitro AP-MS in the absence and presence of RNase A, which revealed that $83 \%$ of PGC- $1 \alpha$ CTD protein-protein interactions rely on RNA (Fig. 2e, Supplementary Fig. 3a, b). Many of the RNA-dependent interacting proteins are involved in skeletal muscle function, but a large number is involved in RNA processing, implying a direct link between the binding of RNAs and splicing factors to the CTD of PGC-1 $\alpha$ (Fig. 2f, Supplementary Fig. 3c). Notably, RNA-dependent interactions also include several TFs (e.g. BTF3 and CEBPG) and components of transcriptional coregulator complexes (e.g. SGF29 and JMJD6) (Fig. 2f). These findings indicate that the CTD of PGC$1 \alpha$ regulates gene transcription and RNA processing in an RNA binding-dependent manner.

\section{PGC-1 $\alpha$ chromatin recruitment is regulated by RNA binding at the CTD}

We next implemented in vitro cross-linking affinity purification followed by sequencing (in vitro uvAP-seq) to investigate the RNA-binding capacity of the PGC-1 $\alpha$ CTD, which revealed direct interactions with a large number of RNAs (Fig. 3a). Interestingly, although PGC-1 $\alpha$ can interact with protein-coding RNAs, the vast majority of bound RNAs were transcribed at regulatory elements such as promoters and distal intergenic regions (Fig. 3b, c), supporting and 
expanding previous reports of non-polyadenylated RNAs binding to PGC-1 $\alpha^{9}$. Motif discovery analysis uncovered 11 motifs enriched among RNAs bound by the CTD (Fig. 3d). Such a diversity of RNA motifs has recently been found to be a common characteristic of a large number of RNA binding proteins (RBPs) $)^{5,12}$, including PGC-1 $\alpha$ FL in primary hepatocytes ${ }^{13}$. Overall, our results now demonstrate that PGC-1 $\alpha$ is a bona fide RBP, with a large promiscuity for binding several types of RNAs harbouring different recognition motifs.

Previous studies have linked PGC-1 $\alpha$ CTD function to RNA processing ${ }^{8}$, e.g. for the stabilization of hepatic metabolic transcripts ${ }^{13}$. Integration of our RNA-seq and in vitro uvAPseq datasets however revealed that over $90 \%$ of protein-coding RNAs bound by PGC-1 $\alpha$ were neither DEG nor alternative spliced, while $97 \%$ of DEG were neither alternative spliced nor bound by PGC-1 $\alpha$ (Fig. 3e). While the small overlap between RNA binding and differential gene expression was also observed in the liver ${ }^{13}$, our results indicate that RNA processing is not the main function of RNA binding to the CTD. Chromatin-bound RBPs have recently emerged as key regulators of gene transcription at active promoters and enhancers ${ }^{12,14}$. Moreover, PGC-1 $\alpha$ activity can be modulated by binding of enhancer and other long noncoding RNAs at DNA regulatory elements ${ }^{9,15}$. A comparison of our RNA binding and PGC$1 \alpha$ FL ChIP-seq data ${ }^{16}$ demonstrates a low correlation between RNA- and chromatin-binding profiles (Supplementary Fig. 4a, b), similar to what has been reported for many other RBPs ${ }^{12,14}$. Thus, RNA interactions with the CTD of PGC-1 $\alpha$ at the site of genomic recruitment, e.g the reported interaction with enhancer $\operatorname{RNAs}^{9}$, seems to be a minor event. Therefore, to define how RNA binding modulates PGC-1 $\alpha$ function, we measured its recruitment to chromatin in the absence and presence of RNase A via subcellular fractionation. This experiment revealed that the absence of nuclear RNAs strongly decreases the amount of chromatin-bound PGC-1 $\alpha$ FL (Fig. 3f). Importantly, subsequent reloading of nuclei with yeast tRNA was sufficient to restore chromatin-bound PGC-1 $\alpha$ FL levels (Fig. 3g). Our data therefore demonstrate that, besides 
regulating protein-protein interactions, RNA binding at the CTD of PGC-1 $\alpha$ is a crucial mechanism controlling its dynamic recruitment to chromatin.

\section{The CTD of PGC-1 $\alpha$ regulates sub-nuclear localization at transcriptional condensates}

While residual levels of PGC-1 $\alpha$ FL in cytoplasm and nucleoplasm of C2C12 myotubes were observed, we found that most of the protein was strongly bound to chromatin (Fig. 4a). In stark contrast, chromatin binding of PGC-1 $\alpha \Delta \mathrm{CTD}$ was markedly reduced, associated with a shift towards higher relative levels in the nucleoplasm and cytoplasm compared to the FL protein (Fig. 4a). Intriguingly, both endogenous and GFP-fused PGC-1 $\alpha$ FL proteins form characteristic nuclear foci, originally defined as nuclear speckles ${ }^{8,15}$. We now observed that deletion of the CTD of GFP-fused PGC-1 $\alpha$ completely abolished its localization in nuclear foci in C2C12 myoblasts (Fig. 4b). Together, our data indicate that a large fraction of PGC-1 $\alpha$ foci represent a chromatin-rich sub-compartment such as chromatin condensates, rather than nuclear speckles that are thought to reside in the nucleoplasm ${ }^{17}$.

Chromatin condensates are membrane-less organelles formed via liquid-liquid phase separation (LLPS) that play a critical regulatory role in gene expression ${ }^{18,19}$. Interestingly, the nuclear foci containing PGC-1 $\alpha$ resemble nuclear condensates formed by $\mathrm{TFs}$ and transcriptional coregulators ${ }^{20,21}$. The formation of liquid-like droplets is often driven by the presence of intrinsically disordered regions (IDR) on proteins and by multivalent protein and RNA interactions of RBPs ${ }^{22}$. PGC-1 $\alpha$ is largely disordered (Fig. 4c, Supplementary Fig. 5a), yet our findings demonstrate that N-terminal IDRs alone are insufficient to mediate the formation of nuclear foci (Fig. 4b). Conversely, in presence of the RRM, PGC-1 $\alpha$ is found in foci (Fig. 4b), implying that RNA binding plays an important role in localising PGC-1 $\alpha$ within nuclear foci. To assess potential liquid-like properties of PGC-1 $\alpha$ foci, we carried out fluorescence recovery after photobleaching (FRAP) analysis that revealed a rapid fluorescence 
recovery characteristic of liquid-like condensates (Fig. 4d, e). We additionally studied the physical properties of PGC-1 $\alpha$ foci by using the aliphatic alcohol 1,6-hexanediol, which resulted in a dissolving of foci within seconds (Fig. 4f, Supplementary Fig. 5b). Finally, the liquid-like properties of PGC-1 $\alpha$-containing condensates was supported by live cell imagingbased detection of fusion events between different foci (Fig. 4g, Supplementary Fig. 5c). While the precise molecular composition of these condensates awaits clarification, it is tempting to speculate that as a strong transcriptional coactivator, PGC-1 $\alpha$ might represent a subcompartment favouring active gene transcription by assembling transcriptional multi-protein complexes. Consistent with this hypothesis, we found that PGC-1 $\alpha$ condensates exhibit an enrichment of Pol 2 phosphorylated at S5 of its CTD (p-Pol 2(S5)) and histone 3 acetylated at K27 (H3K27ac), thus reflecting active transcription at enhancers and promoter (Fig. 4h). Therefore, our findings demonstrate that PGC-1 $\alpha$ condensates compartmentalize the transcriptional machinery to concentrate and stimulate gene transcription at DNA regulatory elements.

Collectively, our results reveal key novel insights into the regulation of complex transcriptional networks, where coregulators have been heralded as master controllers using so far elusive mechanisms ${ }^{23,24}$. By studying PGC-1 $\alpha$ as a prototypical member of the coactivator protein superfamily, we now demonstrate that its highly conserved CTD fulfils a crucial role for controlling gene expression by compartmentalizing multi-protein complexes of transcriptional regulators within nuclear condensates. Remarkably, the assembly of such multi-protein complexes on the chromatin is dynamically regulated by PGC-1 $\alpha$-RNA binding, which might facilitate the spatiotemporal adaptive response to stimuli such as exercise and $\operatorname{diet}^{25}$. These unprecedented mechanistic insights now outline how transcriptional coregulators could interpret environmental cues to control transcriptional networks in a context-specific manner 
via assembly of nuclear condensates containing multi-protein transcriptional complexes and specific DNA regulatory elements.

Physical inactivity, which impairs skeletal muscle metabolism and function, can accelerate the development of a wide spectrum of diseases, including type 2 diabetes, cancer and nonalcoholic fatty liver disease ${ }^{26}$. Modulation of PGC- $1 \alpha$ nuclear condensate formation thus represents an attractive strategy to regulate the function of this transcriptional coactivator. Notably, the formation of abnormal liquid-like condensates has recently been found to be associated with the development of pathologies such as cancer and neurodegenerative diseases $^{27}$. Hence, the fundamental mechanism controlling PGC-1 $\alpha$ function discovered in this work might have important implication in the development of strategies to improve energy metabolism under pathological conditions, e.g. by leveraging pharmacological approaches aimed at the selective modulation of transcriptional condensates ${ }^{28}$. 


\section{Figures}

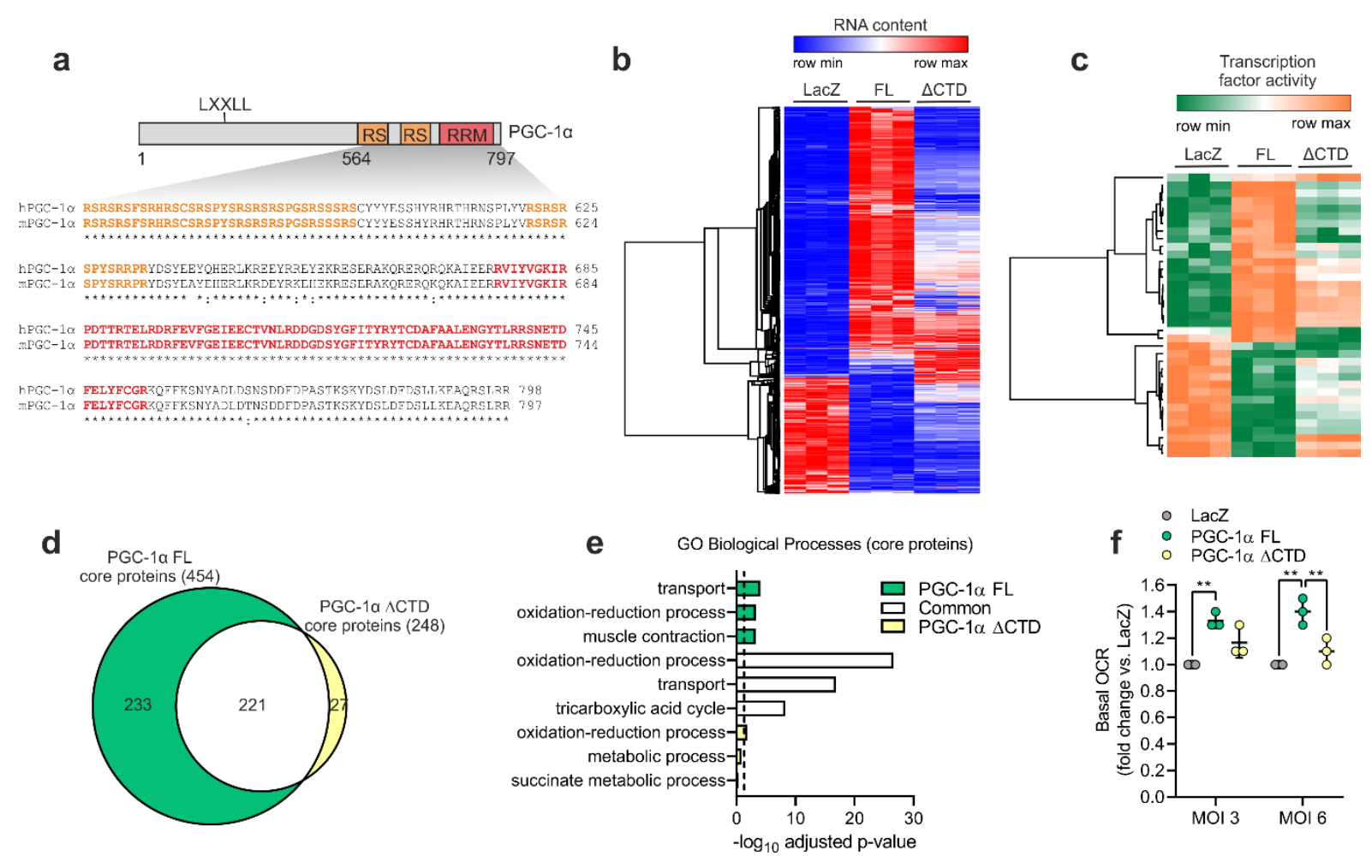

Fig. 1: PGC-1 $\alpha$ function is impaired by deletion of the CTD. a, Illustration of mouse PGC-

$1 \alpha$ (UniProt ID: O70343) with the CTD aligned against the human protein (UniProt ID:

Q9UBK2). b, c, Heat maps with DEG (b) and predicted changes in TF activity (c) induced by overexpression of LacZ (control), PGC-1 $\alpha$ FL or $\triangle \mathrm{CTD}$ in C2C12 myotubes. d, e, Overlap (d) and gene ontology (GO) analysis (e) of core proteins regulated by PGC- $1 \alpha$ FL or $\Delta \mathrm{CTD}$ in C2C12 myotubes. Dashed line represents GO statistical cutoff (adjusted p-value $<0.05$ ). f, Basal oxygen consumption rate (OCR) of $\mathrm{C} 2 \mathrm{C} 12$ myotubes transduced with PGC-1 $\alpha$ FL or $\Delta \mathrm{CTD}$ at two different multiplicity of infections (MOI). Values are mean $\pm \mathrm{SD} ; * * \mathrm{p}<0.01$ 


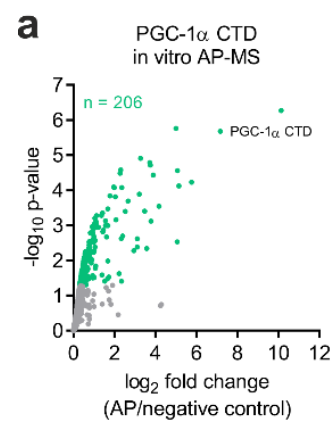

d

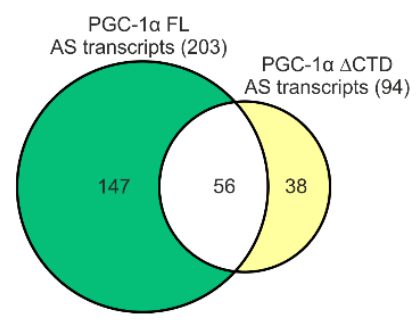

b
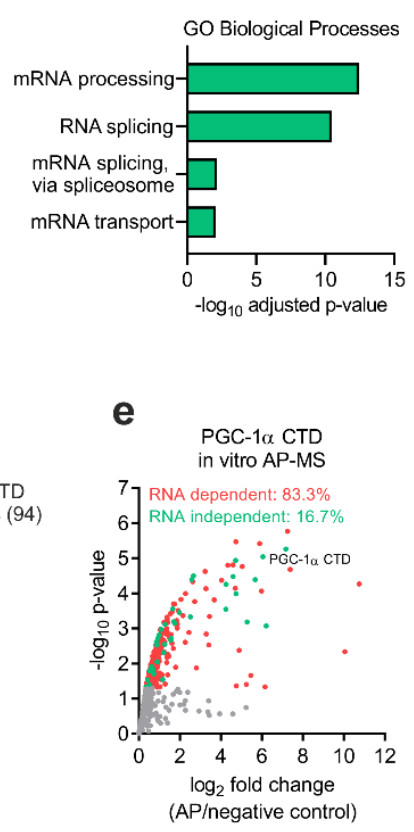
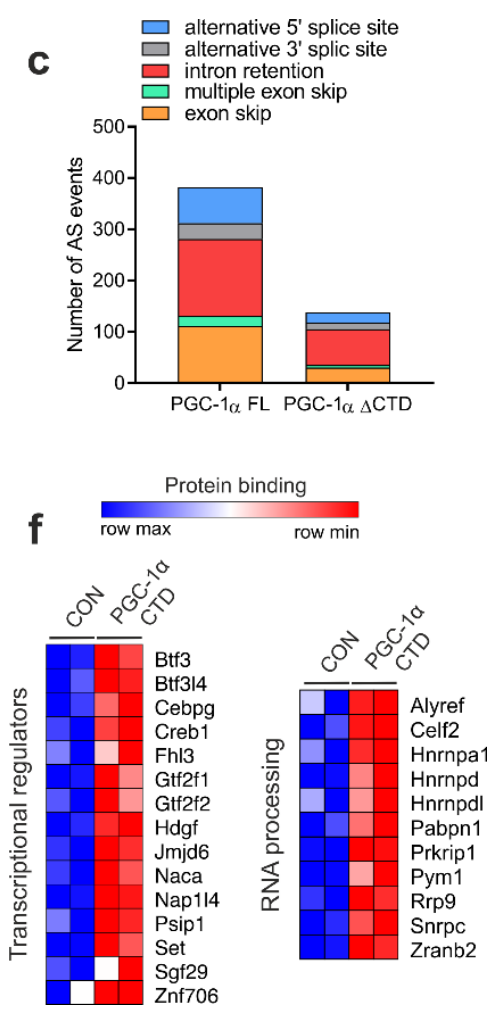

Fig. 2: Most PGC-1 $\alpha$ CTD protein interactions depend on RNA binding. a, Volcano plot with green dots representing proteins interacting with the CTD of PGC-1 $\alpha . \mathbf{b}$, Gene ontology (GO) analysis of PGC-1 $\alpha$ CTD interactome. c, d, Alternative splicing (AS) analysis of transcriptomic data showing the number of AS events (c) and transcripts (d) regulated by PGC$1 \alpha \mathrm{FL}$ or $\triangle \mathrm{CTD}$ in $\mathrm{C} 2 \mathrm{C} 12$ myotubes. e, Volcano plot with red and green dots representing PGC-1 $\alpha$ CTD RNA dependent and independent protein interactions, respectively. f, Heat maps showing PGC-1 $\alpha$ CTD RNA dependent protein interactions with proteins regulating gene transcription and RNA processing. 
a

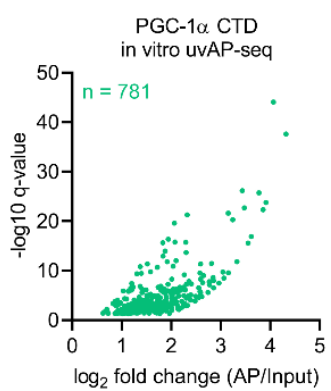

b

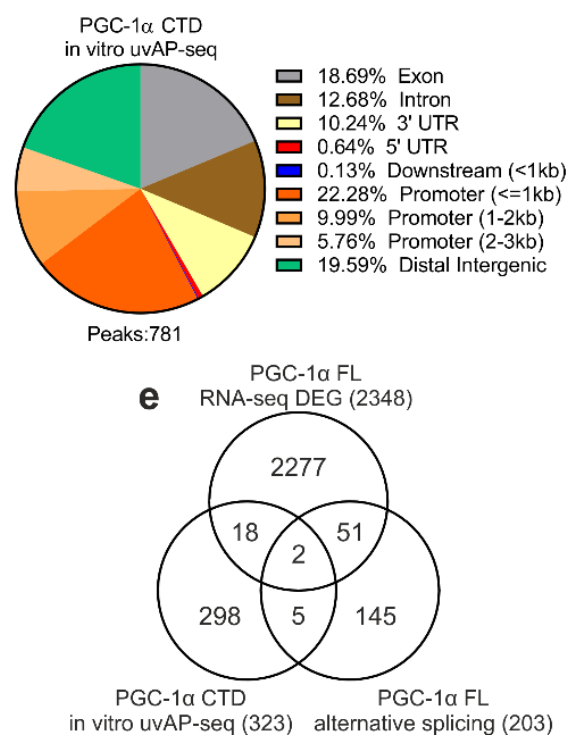

C
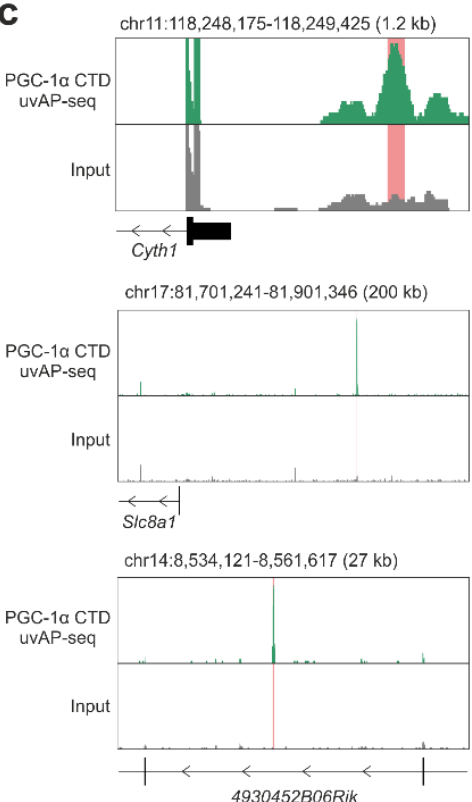

$\mathbf{f}$

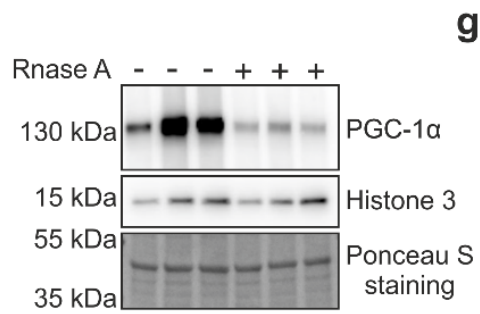

d

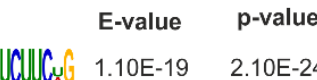

UACABCUU 6.00E-15 $1.10 \mathrm{E}-19$

Co AAGA 2.60E-13 5.00E-18

AUUUAUU 8.40E-13 1.60E-17

UAUAAUA 4.00E-09 7.70E-14

UGGUVUV 1.20E-07 2.30E-12

CCAUdU $1.90 \mathrm{E}-05 \quad 3.60 \mathrm{E}-10$

AAUAAAU 2.30E-05 4.50E-10

AGUCUCA 2.30E-05 4.50E-10

CACGACC 1.50E-02 3.00E-07

CUUAAA 2.80E-02 5.40E-07

Fig. 3: PGC-1 $\alpha$ chromatin recruitment is regulated by RNA binding at the CTD. a,

Volcano plot with green dots representing RNAs interacting with the CTD of PGC-1 $\alpha . \mathbf{b}$, Annotation of in vitro AP-seq peaks representing PGC-1 $\alpha$ CTD bound RNAs. c, Genome browser track view of representative peaks located at promoter (top panel), distal intergenic (middle panel) and intronic regions (bottom panel). d, Motif discovery analysis of PGC-1 $\alpha$ CTD bound RNAs. e, Overlap of transcripts that are differentially expressed (DEG), alternative spliced and bound by PGC-1 $\alpha . \mathbf{f}, \mathbf{g}$, Protein content of PGC- $1 \alpha$ FL bound to chromatin fractions in the absence and presence of $1 \mathrm{mg} / \mathrm{ml}$ of RNase A (f) or following the addition of $5 \mu \mathrm{g} / \mu 1 \mathrm{of}$ yeast tRNA (g). Immunoblots are representative of three independent experiments, each performed in triplicate. Ponceau S staining used as loading control in Fig. $3 \mathrm{f}$ was ran on a different gel. 


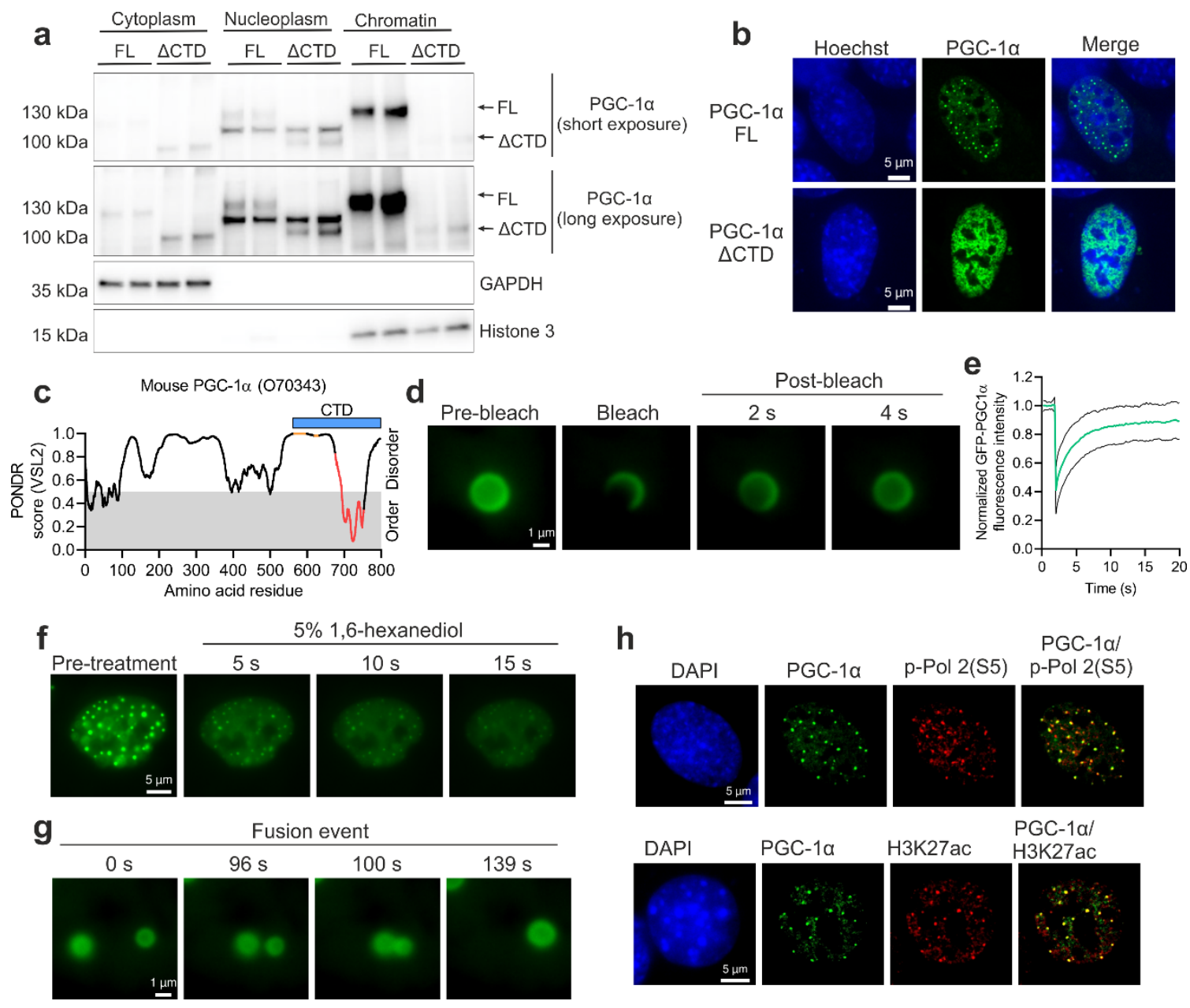

Fig. 4: PGC-1 $\alpha$ activates gene transcription within liquid-like condensates. a, Subcellular fractionation of $\mathrm{C} 2 \mathrm{C} 12$ myotubes expressing PGC-1 $\alpha$ FL or $\Delta \mathrm{CTD}$. b, Nuclear localization of GFP-PGC-1 $\alpha$ FL or $\Delta$ CTD fusion proteins transfected in $\mathrm{C} 2 \mathrm{C} 12$ myoblasts. $\mathbf{c}$, Predictor of Natural Disordered Regions (PONDR) analysis of mouse PGC-1 $\alpha$ protein, with orange dots, red dots and blue bar representing RS domains, RRM and the CTD region under investigation, respectively. d, e, Live-cell imaging (d) and quantification (e) of GFP-PGC-1 $\alpha$ FL FRAP experiments in $\mathrm{C} 2 \mathrm{C} 12$ myoblasts. Green and black lines denote mean and SD of FRAP quantification, respectively. f, Live-cell imaging of 5\% 1,6-hexanediol treatment of $\mathrm{C} 2 \mathrm{C} 12$ myoblasts transfected with GFP-PGC-1 $\alpha$ FL. g, Live-cell imaging of a GFP-PGC-1 $\alpha$ FL droplet fusion event in C2C12 myoblasts. $\mathbf{h}$, Images of GFP-PGC-1 $\alpha$ FL co-stained with DAPI 
bioRxiv preprint doi: https://doi.org/10.1101/2020.09.23.310623; this version posted September 23, 2020. The copyright holder for this preprint (which was not certified by peer review) is the author/funder, who has granted bioRxiv a license to display the preprint in perpetuity. It is made available under aCC-BY-NC-ND 4.0 International license.

(DNA), p-Pol 2(S5) and H3K27ac in C2C12 myoblasts. Immunoblots and microscopy images are representative of at least three independent experiments each in triplicate. 


\section{Supplementary figures}
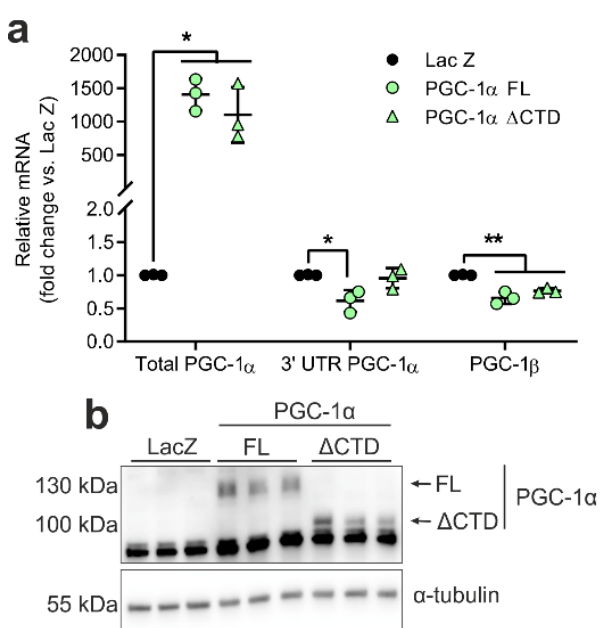

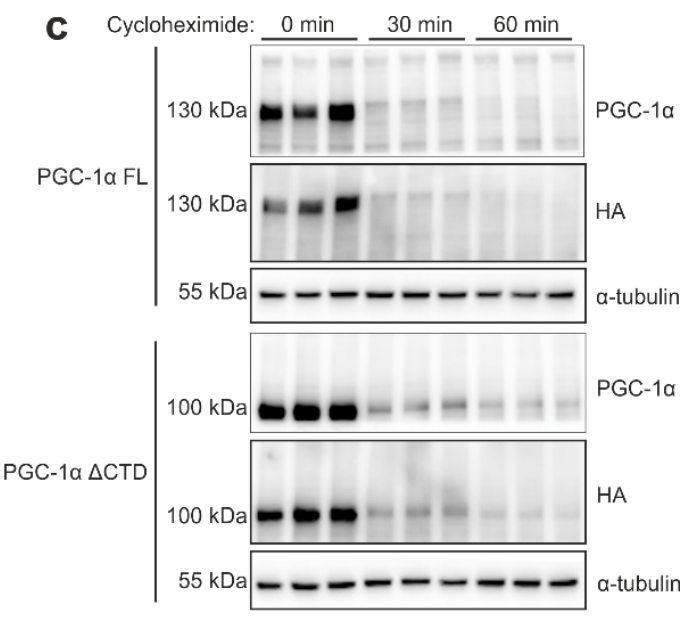

Supplementary Fig. 1: Overexpression of PGC-1 $\alpha$ FL and $\triangle \mathrm{CTD}$ in C2C12 myotubes. a,

b, PGC-1 $\alpha$ mRNA (a) and protein (b) levels in C2C12 myotubes transduced with LacZ (control), PGC-1 $\alpha$ FL or $\Delta$ CTD ( $\mathrm{n}=3$ independent experiments each in triplicate). $\mathbf{c}$, PGC-1 $\alpha$ protein half-life analysis in $\mathrm{C} 2 \mathrm{C} 12$ myotubes transduced with PGC-1 $\alpha$ FL or $\Delta \mathrm{CTD}$ and treated with DMSO as control or $100 \mu \mathrm{g} / \mathrm{ml}$ cycloheximide. Values are mean $\pm \mathrm{SD} ;{ }^{*} \mathrm{p}<0.05$ and ${ }^{* *} \mathrm{p}<0.01$. Immunoblots are representative of three independent experiments, each performed in triplicate. 
a

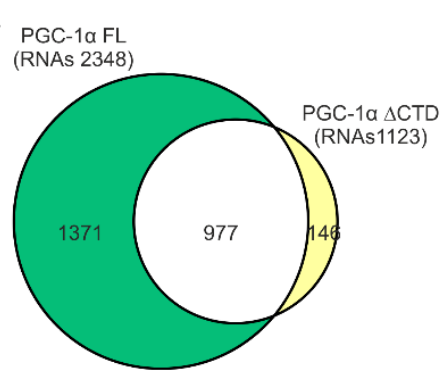

C

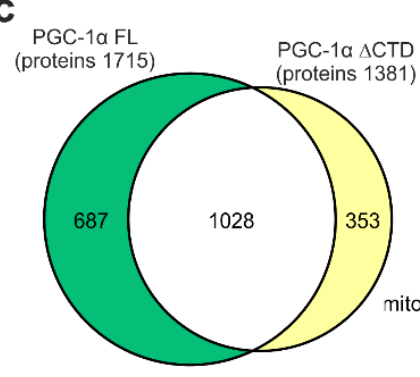

e

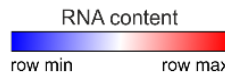

LacZ FL $\triangle$ CTD

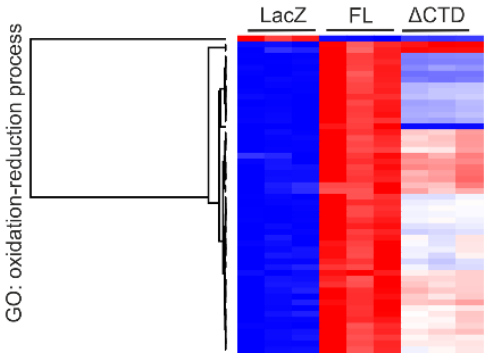

b

GO Biological Processes (transcriptome)

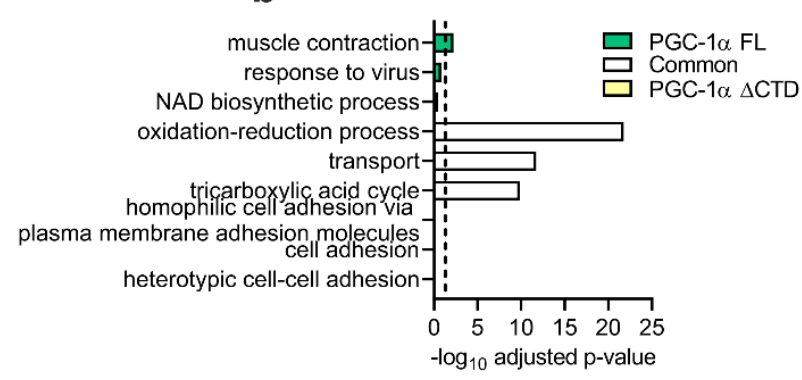

d

GO Biological Processes (proteome)

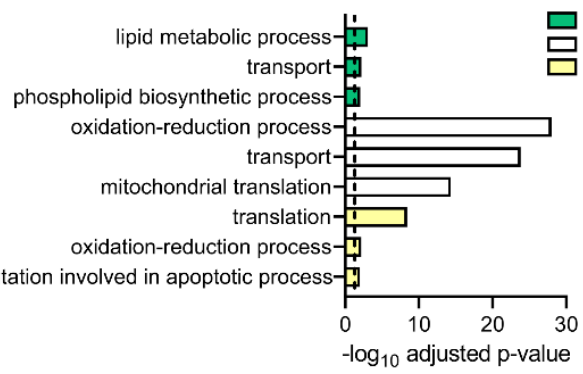

f

Protein content

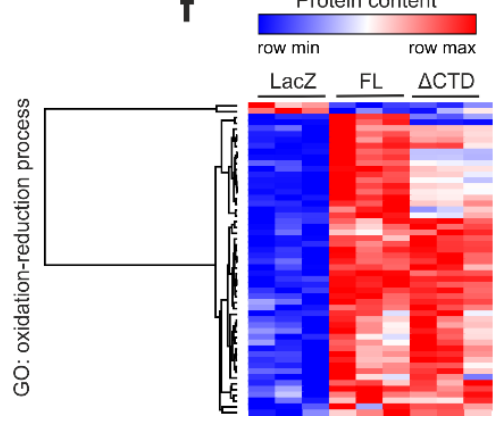

Supplementary Fig. 2: Transcriptome and proteome analysis. a, b, Overlap (a) and gene ontology (GO) analysis (b) of DEG induced by PGC-1 $\alpha$ FL or $\Delta$ CTD in C2C12 myotubes. $\mathbf{c}$, d, Overlap (c) and GO analysis (d) of proteins regulated by PGC-1 $\alpha$ FL or $\Delta$ CTD in C2C12 myotubes. e, f, Heat maps showing the content of RNAs (e) and proteins (f) contained in the GO term oxidation-reduction process in $\mathrm{C} 2 \mathrm{C} 12$ myotubes overexpressing LacZ (control), PGC-1 $\alpha$ FL or $\Delta$ CTD. Dashed line represents GO statistical cutoff (adjusted p-value $<0.05$ ). 

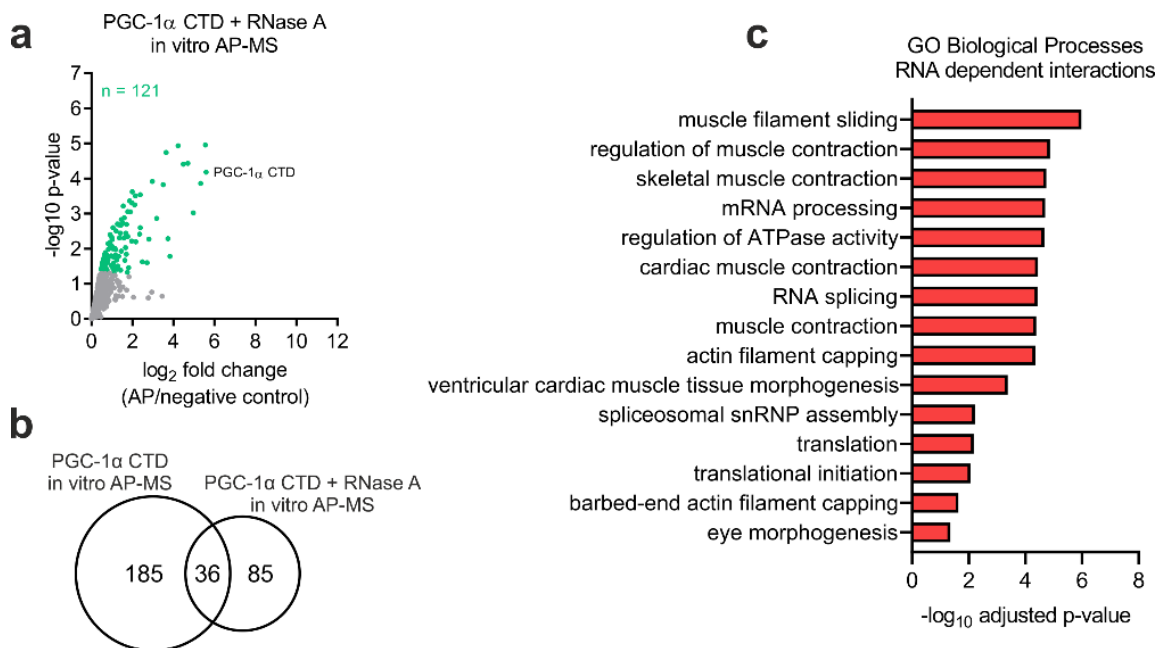

Supplementary Fig. 3: RNA dependent protein-protein interaction analysis. a, Volcano plot with green dots representing proteins interacting with the CTD of PGC-1 $\alpha$ in the presence of $1 \mathrm{mg} / \mathrm{ml}$ of RNase A. b, Overlap of PGC-1 $\alpha$ CTD interacting proteins in the absence and presence of $1 \mathrm{mg} / \mathrm{ml}$ of RNase A. c, Gene ontology (GO) analysis of RNA dependent PGC-1 $\alpha$ CTD interacting proteins. 
a

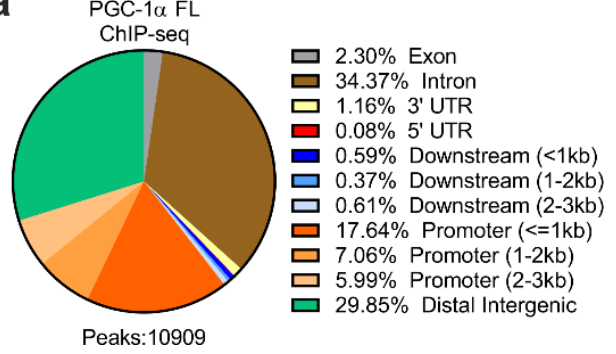

b

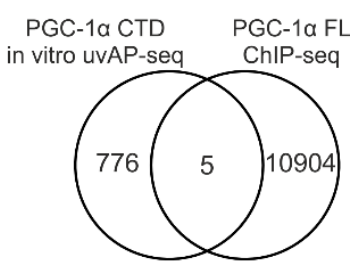

Supplementary Fig. 4: ChIP-seq analysis of PGC-1 $\alpha$ FL. a, Annotation of PGC-1 $\alpha$ FL ChIP-seq peaks. b, Overlap of PGC-1 $\alpha$ CTD and FL in vitro AP-seq and ChIP-seq peaks, respectively. 


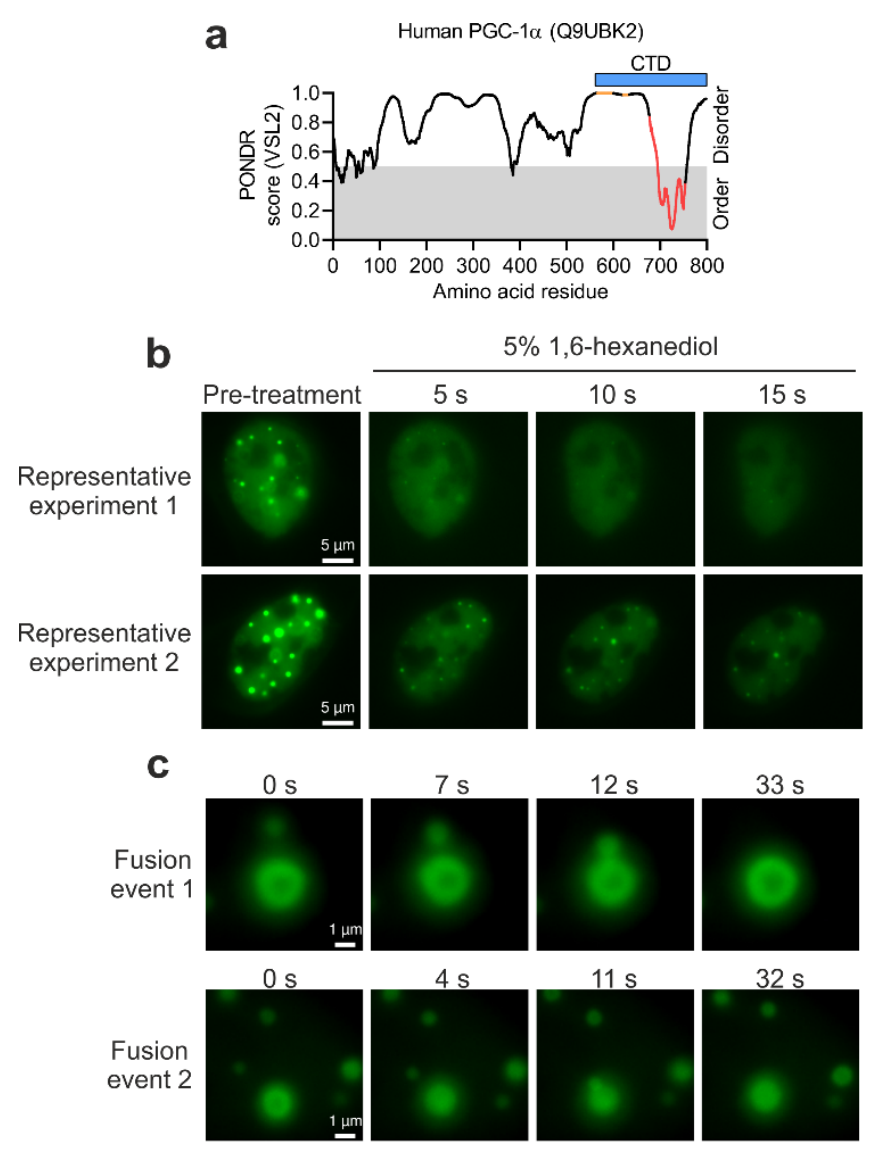

Supplementary Fig. 5: Analysis of liquid-like properties of PGC-1 $\alpha$ nuclear foci. a, Predictor of Natural Disordered Regions (PONDR) analysis of human PGC-1 $\alpha$ protein, with orange and red dots representing RS domains and RRM, respectively. b, Additional live imaging of 5\% 1,6-hexanediol treatment of C2C12 myoblasts transfected with GFP-PGC-1 $\alpha$ FL. c, Additional live imaging of GFP-PGC-1 $\alpha$ FL droplet fusion events in C2C12 myoblasts. Microscopy images are representative of at least three independent experiments, each performed in triplicate. 


\section{References}

1 Lin, J. et al. Transcriptional co-activator PGC-1 alpha drives the formation of slowtwitch muscle fibres. Nature 418, 797-801, doi:10.1038/nature00904 (2002).

2 Puigserver, P. et al. A cold-inducible coactivator of nuclear receptors linked to adaptive thermogenesis. Cell 92, 829-839, doi:10.1016/s0092-8674(00)81410-5 (1998).

3 Yoon, J. C. et al. Control of hepatic gluconeogenesis through the transcriptional coactivator PGC-1. Nature 413, 131-138, doi:10.1038/35093050 (2001).

4 Calvo, J. A. et al. Muscle-specific expression of PPARgamma coactivator-1alpha improves exercise performance and increases peak oxygen uptake. $J$ Appl Physiol (1985) 104, 1304-1312, doi:10.1152/japplphysiol.01231.2007 (2008).

5 Puigserver, P. et al. Activation of PPARgamma coactivator-1 through transcription factor docking. Science 286, 1368-1371, doi:10.1126/science.286.5443.1368 (1999).

6 Wallberg, A. E., Yamamura, S., Malik, S., Spiegelman, B. M. \& Roeder, R. G. Coordination of $\mathrm{p} 300$-mediated chromatin remodeling and TRAP/mediator function through coactivator PGC-1alpha. Mol Cell 12, 1137-1149, doi:10.1016/s10972765(03)00391-5 (2003).

7 Savkur, R. S. \& Burris, T. P. The coactivator LXXLL nuclear receptor recognition motif. J Pept Res 63, 207-212, doi:10.1111/j.1399-3011.2004.00126.x (2004).

8 Monsalve, M. et al. Direct coupling of transcription and mRNA processing through the thermogenic coactivator PGC-1. Mol Cell 6, 307-316, doi:10.1016/s10972765(00)00031-9 (2000).

9 Aguilo, F. et al. Deposition of 5-Methylcytosine on Enhancer RNAs Enables the Coactivator Function of PGC-1alpha. Cell Rep 14, 479-492, doi:10.1016/j.celrep.2015.12.043 (2016). 
10 Balwierz, P. J. et al. ISMARA: automated modeling of genomic signals as a democracy of regulatory motifs. Genome Res 24, 869-884, doi:10.1101/gr.169508.113 (2014).

11 Cho, H. et al. Transcriptional coactivator PGC-1alpha contains a novel CBP80-binding motif that orchestrates efficient target gene expression. Genes Dev 32, 555-567, doi:10.1101/gad.309773.117 (2018).

12 Van Nostrand, E. L. et al. A large-scale binding and functional map of human RNAbinding proteins. Nature 583, 711-719, doi:10.1038/s41586-020-2077-3 (2020).

13 Tavares, C. D. J. et al. Transcriptome-wide analysis of PGC-1alpha-binding RNAs identifies genes linked to glucagon metabolic action. Proc Natl Acad Sci U S A, doi:10.1073/pnas.2000643117 (2020).

14 Xiao, R. et al. Pervasive Chromatin-RNA Binding Protein Interactions Enable RNABased Regulation of Transcription. Cell 178, 107-121 e118, doi:10.1016/j.cell.2019.06.001 (2019).

15 Long, J. et al. Long noncoding RNA Tug1 regulates mitochondrial bioenergetics in diabetic nephropathy. J Clin Invest 126, 4205-4218, doi:10.1172/JCI87927 (2016).

16 Baresic, M., Salatino, S., Kupr, B., van Nimwegen, E. \& Handschin, C. Transcriptional network analysis in muscle reveals AP-1 as a partner of PGC-1alpha in the regulation of the hypoxic gene program. Mol Cell Biol 34, 2996-3012, doi:10.1128/MCB.0171013 (2014).

17 Spector, D. L. \& Lamond, A. I. Nuclear speckles. Cold Spring Harb Perspect Biol 3, doi:10.1101/cshperspect.a000646 (2011).

18 Hnisz, D., Shrinivas, K., Young, R. A., Chakraborty, A. K. \& Sharp, P. A. A Phase Separation Model for Transcriptional Control. Cell 169, 13-23, doi:10.1016/j.cell.2017.02.007 (2017). 
19 Gibson, B. A. et al. Organization of Chromatin by Intrinsic and Regulated Phase Separation. Cell 179, 470-484 e421, doi:10.1016/j.cell.2019.08.037 (2019).

20 Boija, A. et al. Transcription Factors Activate Genes through the Phase-Separation Capacity of Their Activation Domains. Cell 175, 1842-1855 e1816, doi:10.1016/j.cell.2018.10.042 (2018).

21 Sabari, B. R. et al. Coactivator condensation at super-enhancers links phase separation and gene control. Science 361, doi:10.1126/science.aar3958 (2018).

22 Lin, Y., Protter, D. S., Rosen, M. K. \& Parker, R. Formation and Maturation of PhaseSeparated Liquid Droplets by RNA-Binding Proteins. Mol Cell 60, 208-219, doi:10.1016/j.molcel.2015.08.018 (2015).

23 O'Malley, B. W., Qin, J. \& Lanz, R. B. Cracking the coregulator codes. Curr Opin Cell Biol 20, 310-315, doi:10.1016/j.ceb.2008.04.005 (2008).

24 Spiegelman, B. M. \& Heinrich, R. Biological control through regulated transcriptional coactivators. Cell 119, 157-167, doi:10.1016/j.cell.2004.09.037 (2004).

25 Kupr, B. \& Handschin, C. Complex Coordination of Cell Plasticity by a PGC-1alphacontrolled Transcriptional Network in Skeletal Muscle. Front Physiol 6, 325, doi:10.3389/fphys.2015.00325 (2015).

26 Booth, F. W., Roberts, C. K., Thyfault, J. P., Ruegsegger, G. N. \& Toedebusch, R. G. Role of Inactivity in Chronic Diseases: Evolutionary Insight and Pathophysiological Mechanisms. Physiol Rev 97, 1351-1402, doi:10.1152/physrev.00019.2016 (2017).

27 Alberti, S. \& Dormann, D. Liquid-Liquid Phase Separation in Disease. Annu Rev Genet 53, 171-194, doi:10.1146/annurev-genet-112618-043527 (2019).

28 Klein, I. A. et al. Partitioning of cancer therapeutics in nuclear condensates. Science 368, 1386-1392, doi:10.1126/science.aaz4427 (2020). 


\section{Methods}

\section{Cell culture}

C2C12 myoblasts were grown in Dulbecco's modified Eagle's medium (DMEM) supplemented with $10 \%$ fetal bovine serum (growth medium). To induce differentiation, growth medium of about $90 \%$ confluent myoblasts was changed to DMEM supplemented with $2 \%$ horse serum (differentiation medium). Experiments using $\mathrm{C} 2 \mathrm{C} 12$ myotubes were performed after 4 days of differentiation. Cells were maintained at $37^{\circ} \mathrm{C}, 95 \% \mathrm{O}_{2}$ and $5 \% \mathrm{CO}^{2}$.

\section{Generation of DNA plasmids and transfections}

Mouse GFP-PGC-1 $\alpha$ full length (FL) plasmid (Addgene, \#4) was used as template for PCR amplification of a delta C-terminal domain ( $\triangle \mathrm{CTD}$ ) fragment containing amino acid 1-564 of PGC-1 $\alpha$. SalI and BamHI restriction sites were added to the 5' and 3' end of the insert, respectively. The destination plasmid GFP-PGC-1 $\alpha$ FL was digested with SalI and BamHI, following which the PGC-1 $\alpha \Delta$ CTD insert was ligated. Plasmid was corroborated via Sanger sequencing.

Transfection of $\mathrm{C} 2 \mathrm{C} 12$ myoblasts was performed using Opti-MEM ${ }^{\mathrm{TM}}$ (Thermo Fisher Scientific, \#31985070) and Lipofectamine ${ }^{\mathrm{TM}} 2000$ (Thermo Fisher Scientific, \#11668019) following manufacturer's instructions. Twenty four hours after seeding, cells were transfected with $0.5 \mu \mathrm{g}$ of EGFP-PGC-1 $\alpha$ FL (Addgene, \#4) or $\Delta$ CTD for $24 \mathrm{~h}$.

\section{Generation of adenoviral vectors}

Adenovirus vectors were generated with the Adeno-X Adenoviral System 3 following manufacturer's instructions (Takara, \#632267). Briefly, mouse PGC-1 $\alpha$ FL and $\Delta$ CTD were PCR-amplified from the pcDNA-f:PGC1 (Addgene, \# 1026) and pcDNA-f:PGC1(delta CTD) (Addgene, \#1030) plasmid vectors, respectively. N-terminal HA and FLAG tags were 
introduced during PCR amplification, with the amplicon subcloned into the pAdenoXZsGreen1 vector to generate HA-Flag-PGC-1 $\alpha$ FL and $\Delta$ CTD adenoviruses. The $L a c Z$ gene was also subcloned into the pAdenoX-ZsGreen1 vector, which was used to generate a LacZ control adenovirus. All plasmids were corroborated via Sanger sequencing. Adenoviruses were produced and amplified in Adeno-X ${ }^{\mathrm{TM}} 293$ cells (Takara, \# 632271), while titter was determined by fluorescence-activated cell sorting.

\section{Adenovirus transduction}

Cells were transduced with HA-Flag-PGC-1 $\alpha$ FL, $\Delta$ CTD or LacZ adenovirus at multiplicity of infection (MOI) 2-6. Adenoviruses were prepared in the corresponding medium and cells were transduced for $4 \mathrm{~h}$. Next, cells were washed once with phosphate buffered saline (PBS) and, then, incubated in adenovirus-free medium for a total of $48 \mathrm{~h}$.

\section{RNA purification and quantitative PCR (qPCR)}

Cells were collected in TRI Reagent (Sigma \#T9424), following which RNA was purified and reverse transcribed using Direct-zol ${ }^{\mathrm{TM}}$ RNA MiniPrep (Zymo Research, \#R2050) and iScript ${ }^{\mathrm{TM}}$ cDNA Synthesis Kit (Bio-Rad, \#1708891), respectively. Relative changes in mRNA content was quantified by qPCR on a StepOnePlus system (Applied Biosystems) using Fast SYBR ${ }^{\mathrm{TM}}$ Green Master Mix (Thermo Fisher Scientific, \#4385612). The $\Delta \Delta \mathrm{CT}$ method was used for analysis, with TATA binding protein $(T b p)$ as endogenous control.

\section{RNA sequencing (RNA-seq) and alterative splicing analysis}

Libraries were prepared with TruSeq Stranded mRNA Library Kit (Illumina, \#20020595), pairend sequencing was performed using the HiSeq 2500 (Illumina) and data was analysed on the Galaxy platform (https://usegalaxy.eu/). Reads were trimmed with Trim Galore! (Galaxy version 0.4.3.1) and quality was assessed using FastQC (Galaxy version 0.72+galaxy1). Reads were aligned to the mm10 version of the mouse genome using STAR (Galaxy version 2.7.2b), 
while strand specificity and read counting was performed with Infer Experiment (Galaxy version 2.6.4.1) and featureCounts (Galaxy version 1.6.4+galaxy1), respectively. Next, we used DESeq2 (Galaxy version 2.11.40.6) for differential expression analysis (q-value $<0.01$ and fold change $\geq 2$ ) and the resulting data was annotated with Annotate DESeq2/DEXSeq output tables (Galaxy version 1.1.0). Overlap between different datasets was determined with Venny (version 2.1, https://bioinfogp.cnb.csic.es/tools/venny/). Gene Ontology (GO) analysis was performed with DAVID 6.8 (https://david.ncifcrf.gov/), with significance defined as adjusted (Benjamini) p-value $<0.05$. Transcription factors activity analysis was achieved with ISMARA (https://ismara.unibas.ch/mara/), where z-value $>2$ was consider as significant. We used DESeq2 normalized counts to generate heat maps and for hierarchical clustering using Morpheus (https://clue.io/morpheus). Finally, in order to identify alternative splicing events the SplAdder workflow was employed ${ }^{29}$. We produced splicing graphs for all samples belonging to a comparison we were interested in. Afterwards we called splicing events (exon_skip, intron_retention, alt_3prime, alt_5prime, mult_exon_skip) with the SplAdder test procedure using default parameters.

\section{Protein extraction and immunoblotting}

Protein was extracted with protein lysis buffer (50 mM Tris- $\mathrm{HCl}(\mathrm{pH} 7.5), 150 \mathrm{mM} \mathrm{NaCl}, 1$ mM EDTA (pH 8.0), $5 \%$ Glycerol, $1 \%$ NP40, $0.1 \%$ SDS, 1 mM nicotinamide, 1 X Halt ${ }^{\mathrm{TM}}$ Protease Inhibitor Cocktail (Thermo Fisher Scientific, \#87786)) as previously described ${ }^{30}$.

Immunoblotting was performed with precast (Bio-Rad, \#4561096) or home-made SDSpolyacrylamide gels as previously described ${ }^{30}$, and proteins were detected with a primary antibody to PGC-1 $\alpha$ (Santa Cruz Biotechnology, \#sc-518025), $\alpha$-tubulin (Cell Signaling Technology, \#2144S), histone 3 (Abcam, \#ab1791), GAPDH (Cell Signaling Technology, \#2118S) and HA (Sigma, \#11867423001). If required, Ponceau S (Sigma, \#P7170-1L) staining was used as loading control. Secondary antibodies for mouse (Agilent, \#P0260), rabbit 
(Agilent, \#P0399) and rat (Jackson ImmunoResearch, \#112-035-003) were used. Antibody binding was detected using enhanced chemiluminescence HRP substrate detection kit for standard (Thermo Fisher Scientific, \#32106), medium (Thermo Fisher Scientific, \#34076) or high (Thermo Fisher Scientific, \#34095) sensitivity.

\section{Protein half-life}

Protein half-life was measured by treating cells with DMSO as control or $100 \mu \mathrm{g} / \mathrm{ml}$ of cycloheximide (Sigma, \#C4859) for 30 or 60 min. Next, cells were collected for protein extraction and immunoblotting.

\section{Mass spectrometry analysis of whole cell proteome}

Cells were lysed in $80 \mu \mathrm{l}$ of lysis buffer (1\% sodium deoxycholate (SDC), $0.1 \mathrm{M}$ TRIS, $10 \mathrm{mM}$ TCEP, $\mathrm{pH}=8.5$ ) using 10 cycles of sonication (Bioruptor, Diagnode). Samples were reduced for $10 \mathrm{~min}$ at $95^{\circ} \mathrm{C}$ and alkylated at $15 \mathrm{mM}$ chloroacetamide for $30 \mathrm{~min}$ at $37^{\circ} \mathrm{C}$. Proteins were digested by incubation with sequencing-grade modified trypsin (1/50 w/w; Promega,V5113) for $12 \mathrm{~h}$ at $37^{\circ} \mathrm{C}$. Tryptic digests were acidified $(\mathrm{pH}<3)$ using TFA and cleaned up using iST cartridges (PreOmics, P.O.00027) according to the manufacturer's instructions. Samples were dried under vacuum and stored at $-20{ }^{\circ} \mathrm{C}$.

Sample aliquots comprising $25 \mu \mathrm{g}$ of peptides were labelled with isobaric tandem mass tags (TMT 10-plex, Thermo Fisher Scientific, 90110) as described previously ${ }^{31}$. Shortly, peptides were re-suspended in $20 \mu \mathrm{l}$ labelling buffer ( $2 \mathrm{M}$ urea, $0.2 \mathrm{M} \mathrm{HEPES,} \mathrm{pH} 8.3$ ) and $5 \mu \mathrm{L}$ of each TMT reagent were added to the individual peptide samples followed by a $1 \mathrm{~h}$ incubation at $25^{\circ} \mathrm{C}$, shaking at $500 \mathrm{rpm}$. To quench the labelling reaction, $1.5 \mu \mathrm{L}$ aqueous $1.5 \mathrm{M}$ hydroxylamine solution was added and samples were incubated for another 10 min at $25^{\circ} \mathrm{C}$ shaking at $500 \mathrm{rpm}$ followed by pooling of all samples. The $\mathrm{pH}$ of the sample pool was increased to 11.9 by adding $1 \mathrm{M}$ phosphate buffer $(\mathrm{pH} 12)$ and incubated for $20 \mathrm{~min}$ at $25^{\circ} \mathrm{C}$ 
shaking at $500 \mathrm{rpm}$ to remove TMT labels linked to peptide hydroxyl groups. Subsequently, the reaction was stopped by adding $2 \mathrm{M}$ hydrochloric acid until a $\mathrm{pH}<2$ was reached. Finally, peptide samples were further acidified using $5 \%$ TFA, desalted using Sep-Pak Vac 1cc (50 mg) C18 cartridges (Waters, WAT054960) according to the manufacturer's instructions and dried under vacuum.

TMT-labelled peptides were fractionated by high-pH reversed phase separation using a XBridge Peptide BEH C18 column (3,5 $\mu \mathrm{m}, 130 \AA$, 1 mm x 150 mm; Waters, 186003562) on an Agilent 1260 Infinity HPLC system. Peptides were loaded on column in buffer A (20 mM ammonium formate in water, $\mathrm{pH} 10$ ) and eluted using a two-step linear gradient from $2 \%$ to $10 \%$ in 5 minutes and then to $50 \%$ buffer B (20 mM ammonium formate in $90 \%$ acetonitrile, $\mathrm{pH} 10$ ) over 55 minutes at a flow rate of $42 \mu \mathrm{l} / \mathrm{min}$. Elution of peptides was monitored with a UV detector $(215 \mathrm{~nm}, 254 \mathrm{~nm})$ and a total of 36 fractions were collected, pooled into 12 fractions using a post-concatenation strategy as previously described ${ }^{32}$ and dried under vacuum.

Dried peptides were re-suspended in $0.1 \%$ aqueous formic acid and subjected to LC-MS/MS analysis using a Q Exactive HF Mass Spectrometer fitted with an EASY-nLC 1000 (Thermo Fisher Scientific) and a custom-made column heater set to $60^{\circ} \mathrm{C}$. Peptides were resolved using a RP-HPLC column $(75 \mu \mathrm{m} \times 30 \mathrm{~cm})$ packed in-house with C18 resin (ReproSil-Pur C18-AQ, $1.9 \mu \mathrm{m}$ resin; Dr. Maisch, r119.aq.) at a flow rate of $0.2 \mu \mathrm{Lmin}-1$. The following gradient was used for peptide separation: from 5\% B to $15 \%$ B over 10 min to $30 \%$ B over 60 min to $45 \%$ B over 20 min to $95 \%$ B over 2 min followed by 18 min at $95 \%$ B. Buffer A was $0.1 \%$ formic acid in water and buffer B was $80 \%$ acetonitrile, $0.1 \%$ formic acid in water.

The mass spectrometer was operated in DDA mode with a total cycle time of approximately 1 s. Each MS1 scan was followed by high-collision-dissociation (HCD) of the 10 most abundant precursor ions with dynamic exclusion set to $30 \mathrm{~s}$. For MS1, 3e6 ions were accumulated in the 
Orbitrap over a maximum time of $100 \mathrm{~ms}$ and scanned at a resolution of 120,000 FWHM (at $200 \mathrm{~m} / \mathrm{z}$ ). MS2 scans were acquired at a target setting of $1 \mathrm{e} 5$ ions, maximum accumulation time of $100 \mathrm{~ms}$ and a resolution of 30,000 FWHM (at $200 \mathrm{~m} / \mathrm{z}$ ). Singly charged ions and ions with unassigned charge state were excluded from triggering MS2 events. The normalized collision energy was set to $35 \%$, the mass isolation window was set to $1.1 \mathrm{~m} / \mathrm{z}$ and one microscan was acquired for each spectrum.

The acquired raw-files were converted to the mascot generic file (mgf) format using the msconvert tool (part of ProteoWizard, version 3.0.4624 (2013-6-3)) and searched using MASCOT against a murine database (consisting of 49434 forward and reverse protein sequences downloaded from Uniprot on 20141124) and 390 commonly observed contaminants. The precursor ion tolerance was set to $10 \mathrm{ppm}$ and fragment ion tolerance was set to $0.02 \mathrm{Da}$. The search criteria were set as follows: full tryptic specificity was required (cleavage after lysine or arginine residues unless followed by proline), 3 missed cleavages were allowed, carbamidomethylation (C) and TMT6plex (K and peptide $\mathrm{N}$-terminus) were set as fixed modification and oxidation (M) as a variable modification. Next, the database search results were imported into the Scaffold Q+ software (version 4.3.2, Proteome Software Inc.) and the protein false discovery rate was set to $1 \%$ based on the number of decoy hits. Proteins that contained similar peptides and could not be differentiated based on MS/MS analysis alone were grouped to satisfy the principles of parsimony. Proteins sharing significant peptide evidence were grouped into clusters. Acquired reporter ion intensities in the experiments were employed for automated quantification and statistical analysis using a modified version of our in-house developed SafeQuant R script v2.3 $3^{31}$. This analysis included adjustment of reporter ion intensities, global data normalization by equalizing the total reporter ion intensity across all channels, summation of reporter ion intensities per protein and channel, calculation of protein abundance ratios and testing for differential abundance using empirical Bayes 
moderated t-statistics. The calculated p-values were corrected for multiple testing using the Benjamini-Hochberg method, with significance defined as q-value $<0.05$ and fold change $\geq$ 1.2. Overlap between different datasets was determined with Venny (version 2.1, https://bioinfogp.cnb.csic.es/tools/venny/). GO analysis was performed with DAVID 6.8 (https://david.ncifcrf.gov/), with significance defined as adjusted (Benjamini) p-value $<0.05$.

\section{Assessment of oxygen consumption}

Basal oxygen consumption was measured using the Seahorse XF Cell Mito Stress Test Kit (Agilent, \#103015-100) on a Seahorse XF96 Analyzer (Agilent) according to the manufacturer's instructions.

\section{Subcellular fractionation}

Subcellular fractionation was performed as previously described ${ }^{33,34}$, with the following modifications. Cells, on $10 \mathrm{~cm}$ plates, were washed twice with ice-cold PBS and collected into $5 \mathrm{ml}$ of ice-cold PBS. Samples were centrifuged for $2 \mathrm{~min}$ at $500 \mathrm{~g}$ at $4{ }^{\circ} \mathrm{C}$ and the cell pellet was re-suspended in $500 \mu \mathrm{l}$ of ice-cold cytoplasmic lysis buffer $(0.15 \%$ NP-40, $10 \mathrm{mM}$ Tris$\mathrm{HCl}(\mathrm{pH} 7.0), 150 \mathrm{mM} \mathrm{NaCl}$ and $1 \mathrm{X}$ Halt ${ }^{\mathrm{TM}}$ Protease Inhibitor Cocktail (Thermo Fisher Scientific, \#87786)). Following 5 min incubation on ice, samples were homogenized by using a glass Donce homogenizer with 20 strokes with a tight pestle on ice. The resulting cell lysate was layered onto $1250 \mu \mathrm{l}$ of ice-cold sucrose buffer (10 mM Tris- $\mathrm{HCl}(\mathrm{pH} 7.0), 150 \mathrm{mM} \mathrm{NaCl}$, $25 \%$ sucrose and $1 \mathrm{X}$ Halt ${ }^{\mathrm{TM}}$ Protease Inhibitor Cocktail) and centrifuged for $10 \mathrm{~min}$ at 16000 $\mathrm{g}$ at $4^{\circ} \mathrm{C}$. The supernatant containing the cytoplasmic fraction was transferred to a pre-chilled tube and snap frozen in liquid nitrogen. Nuclei pellet was wash once with $1 \mathrm{ml}$ of nuclei wash buffer $\left(0.1 \%\right.$ Triton X-100, 1mM EDTA and $1 \mathrm{X}$ Halt ${ }^{\mathrm{TM}}$ Protease Inhibitor Cocktail in PBS) and centrifuged for $1 \mathrm{~min}$ at $1150 \mathrm{~g}$ at $4^{\circ} \mathrm{C}$. Nuclei pellet was re-suspended in $200 \mu \mathrm{l}$ of glycerol buffer (20 mM Tris- $\mathrm{HCl}$ (pH 8.0), 75 mM NaCl, 0.5 mM EDTA, 50\% glycerol, 0.85 mM DTT 
and 1X Halt ${ }^{\mathrm{TM}}$ Protease Inhibitor Cocktail), after which $200 \mu \mathrm{l}$ of nuclei lysis buffer (1\% NP40, $20 \mathrm{mM}$ HEPES (pH 7.5), $300 \mathrm{mM} \mathrm{NaCl}, 1 \mathrm{M}$ urea, $0.2 \mathrm{mM}$ EDTA, $1 \mathrm{mM}$ DTT and 1X Halt ${ }^{\mathrm{TM}}$ Protease Inhibitor Cocktail) was added. Samples were mixed by pulsed vortex and incubate on ice for $2 \mathrm{~min}$. Following centrifugation for $2 \mathrm{~min}$ at $18500 \mathrm{~g}$ at $4^{\circ} \mathrm{C}$, the supernatant containing the nucleoplasm fraction was transferred to a pre-chilled tube and snap frozen in liquid nitrogen. The chromatin pellet was incubated with $50 \mu \mathrm{l}$ of 1X RQ1 DNase Reaction Buffer with 30U of RQ1 RNase-Free DNase (Promega, \# M6101) for 10 min at $37^{\circ} \mathrm{C}$. Next, samples were placed on ice and $50 \mu \mathrm{l}$ of storage buffer $(10 \mathrm{mM}$ Tris (pH 7.4), $1 \mathrm{mM}$ EDTA, $25 \mathrm{mM} \mathrm{NaCl}, 10 \%$ glycerol and $1 \mathrm{X}$ Halt ${ }^{\mathrm{TM}}$ Protease Inhibitor Cocktail) were added, following which sonication was performed using Bioruptor ${ }^{\circledR}$ Plus (Diagenode) at $4^{\circ} \mathrm{C}$ for 15 min with $30 \mathrm{sec}$ on and $30 \mathrm{sec}$ off. Finally, samples were centrifuged at $16100 \mathrm{~g}$ for $10 \mathrm{~min}$ at $4{ }^{\circ} \mathrm{C}$ and the supernatant containing the chromatin was transferred to a pre-chilled tube and snap frozen in liquid nitrogen. Samples were prepared for immunoblotting as described above.

\section{RNase A and yeast tRNA treatment of nuclei}

Nuclei were isolated as described above, washed once with $1 \mathrm{ml}$ of ice-cold PBS and centrifuged for $1 \mathrm{~min}$ at $1150 \mathrm{~g}$ at $4^{\circ} \mathrm{C}$. The pellet was re-suspended in $500 \mu \mathrm{l}$ of $0.05 \%$ Tween20 in PBS and incubated on ice for 10 min to permeabilize the nuclei. Next, samples were centrifuged for $1 \mathrm{~min}$ at $1150 \mathrm{~g}$ at $4^{\circ} \mathrm{C}$ and washed once with $1 \mathrm{ml}$ of ice-cold PBS. Samples were re-suspended in either $100 \mu \mathrm{l}$ of PBS with or without $1 \mathrm{mg} / \mathrm{ml}$ of RNase A (Sigma, \#R4642) and were incubated for $15 \mathrm{~min}$ at $37^{\circ} \mathrm{C}$. Next, samples were centrifuged at $2300 \mathrm{~g}$ for 10 min at $4^{\circ} \mathrm{C}$ and chromatin fraction was extracted as described above.

For tRNA treatments all samples were digested with RNase A, following which samples were washed twice with $1 \mathrm{ml}$ of ice-cold PBS. Nuclei pellets were re-suspended in $100 \mu \mathrm{l}$ of PBS containing $1 \mathrm{U} / \mu \mathrm{l}$ RNasin ${ }^{\circledR}$ Ribonuclease Inhibitors (Promega, \# N2615) with or without 5 $\mu \mathrm{g} / \mu \mathrm{l}$ of yeast tRNA (Thermo, \#AM7119) and were then incubated for 15 min at $37^{\circ} \mathrm{C}$. 
Subsequently, $1 \mathrm{ml}$ of nuclei wash buffer $\left(0.1 \%\right.$ Triton X-100, $1 \mathrm{mM}$ EDTA and $1 \mathrm{X}$ Halt ${ }^{\mathrm{TM}}$ Protease Inhibitor Cocktail in PBS) was added and samples were centrifuged for 1 min at 1150 $\mathrm{g}$ at $4^{\circ} \mathrm{C}$. Chromatin fraction was extracted as described above.

\section{In vitro affinity purification mass spectrometry (in vitro AP-MS)}

$\mathrm{C} 2 \mathrm{C} 12$ myotubes were transduced with PGC-1 $\alpha$ FL at MOI 3 and nuclear extract was prepared with NE-PER ${ }^{\mathrm{TM}}$ Nuclear and Cytoplasmic Extraction Reagents (Thermo Fisher Scientific, \#78835). Pull-down and negative control comprised $350 \mu \mathrm{g}$ of protein from nuclear extract, while $12 \mu \mathrm{g}$ of recombinant N-terminal His-Tag CTD of mouse PGC-1 $\alpha$ (US Biological Life Sciences, \#156296) was added only to pull-down samples. Samples were incubated overnight at $4^{\circ} \mathrm{C}$ with rotation, following which protein-protein complexes were purified with Pierce $\mathrm{e}^{\mathrm{TM}}$ His Protein Interaction Pull-Down Kit (Thermo Fisher Scientific, \#21277) following manufacturer's instructions. RNA-dependent interactions were assessed by treating nuclear extracts with $1 \mathrm{U} / \mu \mathrm{l}$ RNasin ${ }^{\circledR}$ Ribonuclease Inhibitors (Promega, \# N2615) as control or 1 $\mathrm{mg} / \mathrm{ml}$ of RNase A (Sigma, \#R4642) for $15 \mathrm{~min}$ at $37^{\circ} \mathrm{C}$ before adding the recombinant protein for overnight incubation.

Eluted proteins were incubated with four volumes of $100 \%$ trichloroacetic acid on ice for 10 min. Samples were then centrifuged at $18500 \mathrm{~g}$ for $5 \mathrm{~min}$ and the protein pellet was washed twice with $200 \mu \mathrm{l}$ of cold acetone. The final protein pellet was re-suspended in $40 \mathrm{ul} \mathrm{Gua} \mathrm{buffer}$ (2 M Guanidinium-HCl, 0.1 M Ammonium bicarbonate and $5 \mathrm{mM}$ TCEP), sonicated ten times with Vial Tweeter ultrasonicator (Hielscher) and incubated for $10 \mathrm{~min}$ at $95^{\circ} \mathrm{C}$, followed by alkylation of proteins with $15 \mathrm{mM}$ chloroacetamide for $30 \mathrm{~min}$ at $37^{\circ} \mathrm{C}$. Next, guanidium- $\mathrm{HCl}$ was diluted below $0.4 \mathrm{M}$ with $0.1 \mathrm{M}$ ammonium bicarbonate prior adding $0.5 \mathrm{ug}$ trypsin and incubated for $12 \mathrm{~h}$ at $37^{\circ} \mathrm{C}$ shaking at $300 \mathrm{rpm}$. Tryptic digest was acidified $(\mathrm{pH}<3)$ using TFA and desalted using C18 reverse phase spin columns (Microspin, The Nest Group, Inc., \#SEM 
SS18V) according to the manufacturer's instructions. Peptides were dried under vacuum and stored at $-20^{\circ} \mathrm{C}$.

Dried peptides were resuspended in $0.1 \%$ aqueous formic acid and subjected to LC-MS/MS analysis using a Orbitrap Fusion Lumos Mass Spectrometer fitted with an EASY-nLC 1200 (Thermo Fisher Scientific) and a custom-made column heater set to $60^{\circ} \mathrm{C}$. Peptides were resolved using a RP-HPLC column $(75 \mu \mathrm{m} \times 36 \mathrm{~cm})$ packed in-house with C18 resin (ReproSilPur C18-AQ, $1.9 \mu \mathrm{m}$ resin; Dr. Maisch, r1 19.aq.) at a flow rate of $0.2 \mu \mathrm{Lmin}-1$. The following gradient was used for peptide separation: from $5 \%$ B to $12 \%$ B over 5 min to $35 \%$ B over 40 min to $50 \%$ B over 15 min to $95 \%$ B over 2 min followed by 18 min at $95 \%$ B. Buffer A was $0.1 \%$ formic acid in water and buffer B was $80 \%$ acetonitrile, $0.1 \%$ formic acid in water.

The mass spectrometer was operated in DDA mode with a cycle time of 3 seconds between master scans. Each master scan was acquired in the Orbitrap at a resolution of 120,000 FWHM (at $200 \mathrm{~m} / \mathrm{z}$ ) and a scan range from 375 to $1500 \mathrm{~m} / \mathrm{z}$ followed by MS2 scans of the most intense precursors in the linear ion trap at "Rapid" scan rate with isolation width of the quadrupole set to $1.4 \mathrm{~m} / \mathrm{z}$. Maximum ion injection time was set to $50 \mathrm{~ms}$ (MS1) and $35 \mathrm{~ms}$ (MS2) with an AGC target set to $1 \mathrm{e} 6$ and $1 \mathrm{e} 4$, respectively. Only peptides with charge state $2-5$ were included in the analysis. Monoisotopic precursor selection (MIPS) was set to Peptide, and the Intensity Threshold was set to 5e3. Peptides were fragmented by HCD (Higher-energy collisional dissociation) with collision energy set to $35 \%$, and one microscan was acquired for each spectrum. The dynamic exclusion duration was set to 30 s.

The acquired raw-files were imported into the Progenesis QI software (v2.0, Nonlinear Dynamics Limited), which was used to extract peptide precursor ion intensities across all samples applying the default parameters. The generated mgf-file was searched using MASCOT against a murine database (consisting of 33968 forward and reverse protein sequences downloaded from Uniprot on 20180710), spiked with the sequence of his-tagged Ppargcla 
and 392 commonly observed contaminants using the following search criteria: full tryptic specificity was required (cleavage after lysine or arginine residues, unless followed by proline); 3 missed cleavages were allowed; carbamidomethylation (C) was set as fixed modification; oxidation (M) and acetyl (Protein N-term) were applied as variable modifications; mass tolerance of $10 \mathrm{ppm}$ (precursor) and 0.6 Da (fragments). The database search results were filtered using the ion score to set the false discovery rate to $1 \%$ on the peptide and protein level, respectively, based on the number of reverse protein sequence hits in the dataset. Quantitative analysis results from label-free quantification were processed using the SafeQuant R package v.2.3.2 $2^{31}$ (https://github.com/eahrne/SafeQuant/) to obtain peptide relative abundances. This analysis included global data normalization by equalizing the total peak/reporter areas across all LC-MS runs, data imputation using the knn algorithm, summation of peak areas per protein and LC-MS/MS run, followed by calculation of peptide abundance ratios. Only isoform specific peptide ion signals were considered for quantification. To meet additional assumptions (normality and homoscedasticity) underlying the use of linear regression models and t-Tests, MS-intensity signals were transformed from the linear to the log-scale. The summarized peptide expression values were used for statistical testing of between condition differentially abundant peptides. Here, empirical Bayes moderated t-Tests were applied, as implemented in the

$\mathrm{R} /$ Bioconductor limma package

(http://bioconductor.org/packages/release/bioc/html/limma.html), with significance defined as p-value $<0.05$ and fold change $\geq 1.2$. Overlap between different datasets was determined with Venny (version 2.1, https://bioinfogp.cnb.csic.es/tools/venny/). GO analysis was performed with DAVID 6.8 (https://david.ncifcrf.gov/), with significance defined as adjusted (Benjamini) p-value $<0.05$. Heat maps were generated with Morpheus (https://clue.io/morpheus).

\section{In vitro UV crosslinking affinity purification sequencing (in vitro uvAP-seq)}


Cells were transduced as for in vitro AP-MS. Total RNA was then extracted with Direct-zol ${ }^{\mathrm{TM}}$ RNA MiniPrep kit (Zymo Research, \#R2050) and $5 \mu \mathrm{g}$ of RNA were used for input, pull-down or negative control samples. Pull-down samples were prepared by incubating $5 \mu \mathrm{g}$ of RNA with $12 \mu \mathrm{g}$ of recombinant N-terminal His-Tag CTD of mouse PGC-1 $\alpha$ (US Biological Life Sciences, \#156296) for $30 \mathrm{~min}$ at $37^{\circ} \mathrm{C}$ with shaking at $500 \mathrm{rpm}$ in $500 \mu 1$ of PBS with RNasin ${ }^{\circledR}$ Ribonuclease Inhibitors (Promega, \#N261B). Negative control samples comprised $5 \mu \mathrm{g}$ of RNA combined with HisPur Cobalt Resin (see details below), which were incubated as described for pull-down samples. RNA-protein interactions were crosslinked by UV irradiating ( $5 \mathrm{~mJ} / \mathrm{cm}^{2}$ at $250 \mathrm{~nm}$ UV wavelength) opened tubes with samples on ice, following which PBS with RNasin ${ }^{\circledR}$ Ribonuclease Inhibitors was added to $1 \mathrm{ml}$ final volume. RNA-protein complexes were purified with Pierce ${ }^{\mathrm{TM}}$ His Protein Interaction Pull-Down Kit (Thermo Fisher Scientific, \#21277) with the following modifications. HisPur Cobalt Resin was washed 5 times with wash solution and once with PBS in a $1.5 \mathrm{ml}$ tube. Crosslinked samples were combined with HisPur Cobalt Resin and were incubated for 1 hour at $4{ }^{\circ} \mathrm{C}$ with rotation in $1.5 \mathrm{ml}$ tubes. Following incubation, HisPur Cobalt Resin containing the immobilized bait protein-RNA complexes were washed 5 times with wash buffer by centrifugation at $1250 \times \mathrm{g}$ for $1 \mathrm{~min}$. RNA was then partially digested by re-suspending washed HisPur Cobalt Resin in $1 \mathrm{ml}$ of PBS containing $10 \mu \mathrm{l}$ of 1:1500 diluted Ambion ${ }^{\mathrm{TM}}$ RNase I (Thermo Fisher Scientific, \#AM2294), following incubation at $37^{\circ} \mathrm{C}$ for 3 min with shaking at $700 \mathrm{rpm}$ and then washed 5 times as described above. Subsequently, DNA was digested by incubating pull-down, negative control and input samples for $30 \mathrm{~min}$ at $37^{\circ} \mathrm{C}$ with shaking at $800 \mathrm{rpm}$ with $0.05 \mathrm{U} / \mu 1$ of RQ1 RNaseFree DNase in 1X RQ1 RNase-Free DNase Reaction Buffer (Promega, \# M6101). RNA was eluted from HisPur Cobalt Resin by incubating samples in a final volume of $100 \mu 1$ of PBS with $0.4 \mathrm{mg} / \mathrm{ml}$ of Proteinase K (Macherey-Nagel, \#740506) and incubated for $2 \mathrm{~h}$ at $65^{\circ} \mathrm{C}$ 
with shaking at $800 \mathrm{rpm}$. Finally, RNA was purified with Direct-zol ${ }^{\mathrm{TM}}$ RNA MiniPrep kit and stored at $-80^{\circ} \mathrm{C}$.

Purified RNA from input and pull-down samples was used to prepare libraries with Stranded Total RNA Prep with Ribo-Zero Plus kit (Illumina, \# 20040525). Of note, the amount of RNA recovered from negative control samples was not sufficient for library preparation. Single-end sequencing was performed using the NextSeq 500 (Illumina) and data was analysed on the Galaxy platform (https://usegalaxy.eu/). Reads were trimmed with Trim Galore! (Galaxy version 0.4.3.1) and quality was assessed using FastQC (Galaxy version 0.72+galaxy1). Reads were aligned to the mm10 version of the mouse genome using STAR (Galaxy version 2.7.2b) and duplicated reads were removed with MarkDuplicates (Galaxy version 2.18.2.2). Next, we used MACS2 callpeak (Galaxy version, \#2.1.1.20160309.6) for peak calling (q-value $<0.05$ and fold change $\geq 1.5$ ), while ChIPseeker (Galaxy version, \#1.18.0+galaxy1) was used to annotate peaks. FASTA files from significant peaks were generated with Extract Genomic DNA (Galaxy version, \#3.0.3) and DNA was converted to RNA with RNA/DNA (Galaxy version, \# 1.0.2). FASTA files were used for motif discovery with DREME (Version 5.1.1, http://meme-suite.org/), with E-value threshold $<0.05$. Normalized BAM files were generated with bamCoverage (Galaxy version 3.0.2.0) and data was visualized on the Integrated Genome Browser-9.1.4 $4^{35}$ to generate representative genome browser figures. The overlap between in vitro uvAP-seq and PGC-1 $\alpha$ ChIP-seq data (GEO accession: GSE51178) was performed with bedtools Intersect intervals (Galaxy version, \#2.29.0), while overlap with RNA-seq and alternative splicing data was defined with Venny (version 2.1, https://bioinfogp.cnb.csic.es/tools/venny/).

\section{Confocal microscopy}

Cells were seeded at $2 \times 10^{4}$ cells per well on glass coverslips in 24 well plates and transfected as described above. Cells were fixed with $4 \%$ formaldehyde in PBS for 15 min, washed three 
times with PBS and incubated for 15 min with $1 \mu \mathrm{g} / \mathrm{ml}$ of Hoechst 33342 (Thermo Fisher Scientific, \#H3570) in PBS. Next, cells were washed three times with PBS and mounted in 5 $\mu 1$ of ProLong ${ }^{\text {TM }}$ Gold Antifade Mountant (Thermo Fisher Scientific, \#P36930).

For immunofluorescence staining, after fixation, cells were permeabilized with $0.1 \%$ Triton X100 in PBS for 5 min, washed three times with PBS and blocked with $10 \%$ goat serum in PBS for $30 \mathrm{~min}$. Next, cells were incubated for $1 \mathrm{~h}$ with primary antibodies (dilution in $10 \%$ goat serum in PBS) to p-Pol 2(S5) (1:1000 dilution; Abcam, \#ab5408) and H3K27ac (1:2000; Abcam, \#ab4729). Following three washes with PBS, cells were incubated for $1 \mathrm{~h}$ with secondary antibodies (1:1000 dilution in 10\% goat serum in PBS) conjugated to Alexa Fluor 568 (Thermo Fisher Scientific, \#A-21124 or \#A-11011). Cells were then washed three times with PBS and mounted in $5 \mu 1$ of ProLong ${ }^{\mathrm{TM}}$ Gold Antifade Mountant with DAPI (Thermo Fisher Scientific, \# P36931). All immunofluorescence staining steps were performed at room temperature.

Samples were imaged with an Olympus SpinSR CSU-W1 confocal microscope equipped with UPLAPO 100XOHR objective lens and Hamamatsu Flash4 V3 sCMOS camera. Z-stack images at 100X magnification were taken using Olympus cellSens Dimension software.

\section{Live-cell imaging}

Cells were seeded at $2 \times 10^{4}$ cells per chamber on an 8 well chamber slides (ibidi, \#80826) and transfected as described above. Live-cells imaging was performed using FEI MORE microscope, with cells maintained at $37^{\circ} \mathrm{C}$ and $5 \% \mathrm{CO}_{2}$. As light source for GFP imaging a spectraX LED with 470/24 nm excitation and 517/20 nm emission filter was used. Images were recorded with Hamamatsu OCRA flash 4.0 cooled sCMOS at 100X (numerical aperture 1.4, oil) magnification with Live Acquisition 2.5 software, while post-processing and 
deconvolution were carried out with Fiji 1.52p and Huygens Professional 19.10 software, respectively.

Cells were imaged before and after treatment with 5\% 1,6-Hexanediol (Sigma, \#240117) diluted in growth medium for up to $60 \mathrm{~s}$.

Fluorescence recovery after photobleaching (FRAP) was performed with $488 \mathrm{~nm}$ laser and $470 / 24 \mathrm{~nm}$ filter with laser power set to $100 \%$ (Dwell time: $0.962 \mathrm{~ms} / \mu \mathrm{m} 2$, line overlapping 75\%, ROI loop count: 10, exposure time: $20 \mathrm{~ms}$, cycle time: $185 \mathrm{~ms}$ ). Ten images were obtained pre-bleaching, while 399 images were obtained every $185 \mathrm{~ms}$ post-bleaching. Intensities of FRAP regions were extracted with Fiji 1.52p software tool. FRAP Data were background corrected and analysed with the online tool EasyFRAP-web (https://easyfrap.vmnet.upatras.gr/).

\section{Chromatin immunoprecipitation sequencing (ChIP-seq) analysis}

Previously published PGC-1 $\alpha$ ChIP-seq data (GEO accession: GSE51178) ${ }^{36}$ was analysed on the Galaxy platform (https://usegalaxy.eu/) for comparison with the datasets generated in this study. Reads were trimmed with Trim Galore! (Galaxy version 0.4.3.1) and quality was assessed using FastQC (Galaxy version 0.72+galaxy1). Reads were aligned to the mm10 version of the mouse genome using Bowtie2 (Galaxy Version 2.3.4.3+galaxy0) and low quality reads (phred $<20$ ) were filtered out with Filter (Galaxy Version 2.4.1). Next, we used MACS2 callpeak (Galaxy version, \#2.1.1.20160309.6) for peak calling (q-value $<0.05$ and fold change $\geq 2$ ), while ChIPseeker (Galaxy version, \#1.18.0+galaxy1) was used to annotate peaks. Normalized BAM files were generated with bamCoverage (Galaxy version 3.0.2.0).

\section{Statistics and reproducibility}

All qPCR, immunoblotting, confocal microscopy and live-imaging experiments were performed at least three independent times with similar results. FRAP quantification was 
performed with a total of 50 foci from independent experiments. Number of replicates per experiment is indicated in the figure legend when appropriate.

For qPCR and oxygen consumption data analysis, statistical significance was determined with one-way ANOVA with Tukey’s post hoc test using GraphPad Prism (v.8.0). Significance was considered with a $\mathrm{p}$-value $<0.05$. Values are expressed as mean $\pm \mathrm{SD}$.

RNA-seq, ChIP-seq, mass spectrometry, in vitro AP-MS and in vitro AP-seq experiments were performed once with two or three biological replicates as indicated in figure legends. Statistical analysis of these experiments is described above in their corresponding sections.

\section{Data availability}

RNA-seq and in vitro AP-seq data will be deposited to the Gene Expression Omnibus. Whole proteome analysis and in vitro AP-MS will be deposited to PRIDE. All other data are available from the corresponding author upon reasonable request.

\section{References}

29 Kahles, A., Ong, C. S., Zhong, Y. \& Ratsch, G. SplAdder: identification, quantification and testing of alternative splicing events from RNA-Seq data. Bioinformatics 32, 18401847, doi:10.1093/bioinformatics/btw076 (2016).

30 Perez-Schindler, J., Summermatter, S., Santos, G., Zorzato, F. \& Handschin, C. The transcriptional coactivator PGC-1alpha is dispensable for chronic overload-induced skeletal muscle hypertrophy and metabolic remodeling. Proc Natl Acad Sci U S A 110, 20314-20319, doi:10.1073/pnas.1312039110 (2013).

31 Ahrne, E. et al. Evaluation and Improvement of Quantification Accuracy in Isobaric Mass Tag-Based Protein Quantification Experiments. J Proteome Res 15, 2537-2547, doi:10.1021/acs.jproteome.6b00066 (2016). 
32 Wang, Y. et al. Reversed-phase chromatography with multiple fraction concatenation strategy for proteome profiling of human MCF10A cells. Proteomics 11, 2019-2026, doi:10.1002/pmic.201000722 (2011).

33 Mayer, A. \& Churchman, L. S. A Detailed Protocol for Subcellular RNA Sequencing (subRNA-seq). Curr Protoc Mol Biol 120, 429 21-24 29 18, doi:10.1002/cpmb.44 (2017).

34 Kustatscher, G., Wills, K. L., Furlan, C. \& Rappsilber, J. Chromatin enrichment for proteomics. Nat Protoc 9, 2090-2099, doi:10.1038/nprot.2014.142 (2014).

35 Freese, N. H., Norris, D. C. \& Loraine, A. E. Integrated genome browser: visual analytics platform for genomics. Bioinformatics 32, 2089-2095, doi:10.1093/bioinformatics/btw069 (2016).

36 Baresic, M., Salatino, S., Kupr, B., van Nimwegen, E. \& Handschin, C. Transcriptional network analysis in muscle reveals AP-1 as a partner of PGC-1alpha in the regulation of the hypoxic gene program. Mol Cell Biol 34, 2996-3012, doi:10.1128/MCB.0171013 (2014).

Acknowledgements We thank C. Beisel (Genomics Facility Basel, ETH Zürich), P. Demougin (Life Sciences Training Facility, Biozentrum) and K. Schleicher (Imaging Core Facility, Biozentrum) for technical help. This work was supported by the Novartis Foundation for Medical-Biological Research (J.P.S), Research Fund of the University of Basel (J.P.S), the Swiss National Science Foundation, the European Research Council (ERC) Consolidator grant 616830-MUSCLE_NET, Swiss Cancer Research grant KFS-3733-08-2015, the Swiss Society for Research on Muscle Diseases (SSEM), SystemsX.ch, the Novartis Stiftung für Medizinisch-Biologische Forschung and the University of Basel (C.H.). 
Author Contributions: JPS and CH conceived, designed and supervised the study. JPS, BK, KSH, VA, JD, GM, EVF, BKC, DR and AS performed experiments. JPS, KSH, BK, VA, TS, $\mathrm{DR}, \mathrm{AS}, \mathrm{MH}, \mathrm{SH}$ and $\mathrm{CH}$ performed data analysis and interpretation. JPS and $\mathrm{CH}$ wrote the manuscript. All authors reviewed the manuscript.

Competing interest The authors declare no competing interests. 